\title{
Artificial Companions in Stroke Rehabilitation: Likeability, Familiarity and Expectations
}

\author{
Marylyn Alex \\ University of Auckland \\ male962@aucklanduni.ac.nz
}

\author{
Danielle Lottridge \\ University of Auckland \\ d.lottridge@auckland.ac.nz
}

\author{
Burkhard C. Wünsche \\ University of Auckland \\ burkhard@cs.auckland.ac.nz
}

\begin{abstract}
There is a growing interest in digital companionship and artificial companions (ACs) as they are introduced in rehabilitation and healthcare services for the elderly and people with disabilities. We conduct an exploratory, pre-adoption study to better understand first impressions and likeability of ACs with older individuals in a stroke rehabilitation context. We interviewed 11 participants with stroke-related impairments as they viewed depictions of ACs and engaged in interactive gameplay. We found two main axes in which participants judge ACs' likeability: familiarity and expectations, where more familiarity and having expectations were associated with likeability. We relate these findings to literature on ACs for health promotion for survivors of stroke and discuss implications for the design of ACs.
\end{abstract}

\section{Introduction}

Digital companions are being issued by insurance companies and used by real patients as modernized healthcare services explore ways to increase their reach [1]. Digital companions or Artificial Companions (ACs) are being incorporated into healthcare services for the elderly and people with disabilities to support social interaction and physical and emotional well-being. These types of support are especially important for survivors of stroke-the most common cause of adult disability worldwide [2]-where social isolation exacerbates outcomes [3]. There is a tremendous need to support stroke recovery with technology [4], however, usage of technological support is low: a survey conducted in 2018 found that approximately $50 \%$ of stroke rehabilitation therapists have used technologies such as virtual reality video games with movement sensors such as Wii or Kinect and they only use them with patients on average once per week [5].

Stroke rehabilitation therapy may benefit from companionship. Disabilities from stroke impair both cognitive and physical function [6] and healing from stroke requires sustained engagement in rehabilitation therapy, which requires high motivation [7]. Conventional stroke rehabilitation therapy is perceived as being tedious, less effective and discouraging due to its repetitive nature [8, 9, 10]. The lack of motivation and adherence to stroke rehabilitation therapy is associated to poorer outcomes [11, 12, 13, 14, 15]. Social isolation contributes to lack of motivation and adherence to stroke rehabilitation therapy [16, 17, 18, 19].

Companionship combats social isolation and fosters mental and physical well-being [20, 21]; companionship can be supported by pets and pet therapy [22] [23] as well as robots [24, 25]. Older adults and people with disabilities, such as those who have had a stroke, face greater barriers in receiving companionship [26, 27, 28]. Research indicates there is potential for people to derive companionship from ACs [29, 30], but we know relatively little about how older adults and people with disabilities perceive ACs in a stroke rehabilitation context. Companions in the forms of a robot cat (e.g., [31]), dog (e.g., [32]), seal (e.g., [33]), and other creatures such as Tamagotchi (e.g., [34]) have been evaluated but not in the context of stroke rehabilitation. A dolphin Bandit was created for the stroke context [35], but not yet formally evaluated. It is important to evaluate ACs in the stroke rehabilitation context. This study takes a step toward addressing this gap.

To investigate first impressions of ACs in stroke rehabilitation, we conducted an exploratory study with survivors of stroke in a creative rehabilitation programme. We interviewed older adults who were stroke survivors using depictions of ACs and an interactive game. We framed our questions in terms of likeability to encourage participants to share their own thoughts and feelings. Thematic analysis found that participants focused on familiarity of ACs when explaining likeability, where more familiar ACs were more likeable. Further, familiarity enabled participants 
to form expectations regarding $\mathrm{AC}$ interactions, which was also key for likeability. We discuss the implications of these findings for the design of ACs in stroke rehabilitation.

\section{Related work}

In this section, we review the published literature on ACs, in particular those targeted toward providing companionship to older adults and those with disabilities in a healthcare context.

\subsection{Artificial companions: virtual pets, robots, avatars, etc.}

ACs such as robot pets are gaining increasing interest as social companions. Early research suggested that such interventions might work well for particular target groups or application scenarios, including autistic children or intensive care units where no living animals are allowed [36].

A popular example of a robot companion for elderly people is Paro, a robotic seal. Studies showed psychosocial benefits for nursing home residents [37].

Coghlan et al. [38] caution that disadvantages and ethical issues with robotic pets are not yet well understood. For example, a robotic pet might cause emotional stress if it malfunctions, might create a sense of failure if users cannot interact with it as expected, and might not offer the same meaningful experience as real pets. There might also be privacy and security concerns [39]. However, Coghlan et al. conclude that while robot pets cannot simply replace living animals, they have other advantages, which may help with improving physical and mental health [38]. Examples are greater accessibility (lower costs over a lifetime than, say, a real dog; suitable even for small living spaces or hospital beds; no regular care being required (food, grooming)) and the possibility of adding extra functionality. Coeckelbergh suggests users empathise with robots and try to protect them from "suffering" [40].

Kriglstein and Wallner introduced a dog prototype to elderly participants and concluded that simplicity and friendliness were important [32]. Further, a new companion robot Lovot [41] was intentionally designed with less functionality in order to appear more childlike, non-threatening and needy. As of the writing of this article, there was no academic research on the acceptability of Lovot.

Others have investigated what qualities lead to enjoyment and trust. Dragone et al. [42] created a combination of physical robots and virtual characters into a Mixed Reality Agent (MiRA) using a Mixed
Reality visualisation. The agent and the robot followed voice instructions given by humans. Most participants gave positive feedback and enjoyed the experience overall. Another study explored how avatars can be designed to provide positive behavioral change. It was concluded that facial expression and personality of the avatars are important in establishing trust [43]. A similar study investigating trust in robots (rather than avatars) found that human characteristics of the robot (e.g. personality) have only a small effect, and robot characteristics (e.g., performance, appearance, proximity) are most important in trust development [44].

Virtual pets such as Tamagotchi and Digimon were introduced in the $1990 \mathrm{~s}$ as simple virtual pets on a small keychain interface. They share several behaviours with other ACs such as robot companions (e.g., Aibo the robot dog launched by Sony, iCat the robot cat launched by Philips, Tama the robot that resembles a face launched by JSK Laboratories, and NeCoro the robot cat launched by Omron) as they all react positively towards frequent user interaction. People can become very attached to Tamagotchi and committed to taking care of them [23]. Luh et al. [45] developed a companionship scale for artificial pets. The authors report that the key companionship factors were enjoyment, responsibility, and psychological satisfaction, where the latter one had the highest impact. The authors define psychological satisfaction as satisfying various needs, thereby providing a sense of security and belongingness, friendship and personal identity. The authors report that users associated psychological satisfaction with experiencing attachment, self-disclosure, feelings of trust, love, belongingness, and a sense of reality. Virtual pets can provide companionship and teach children about responsibility and empathy [34, 46]. To date, there is little research investigating older adults' impressions of such virtual pets in a stroke rehabilitation context, which is a gap that we address in our research. We draw from the literature to select ACs to investigate, namely Tamagotchi, and a virtual dog and cat based on Aibo, iCat, and NeCoro.

\subsection{Artificial companions in rehabilitation}

Most research on ACs in rehabilitation focuses on robot pets developed and deployed to offer companionship for older adults [38]. Heerink et al. [31] developed an extension of the Unified Theory of Acceptance and Use of Technology (UTAUT) for social robots by older adults, which they tested with 4 week-long experiments with the iCat robot and the roboCare interface. Their model accounted for on 
average $69 \%$ of the variance in usage intentions and on average $54 \%$ of the variance in actual use. A 2019 systematic analysis on social robots for the older adults indicated positive impacts on agitation, anxiety, and quality of life but no statistical significance was found in the meta-analysis [47].

Zora is a humanoid companion robot [48] approximately the size of a baby, that has been used with dementia patients [49]. Zora encourages patients to exercise, interacts through conversation, plays games and cuddles, with the aim to help patients to be motivated and sociable. Paro the robot seal has been studied extensively in dementia care. Paro can provide psychosocial benefits as well as improving social outcomes for dementia patients [33, 37]. Patients with Down syndrome, Hanhart syndrome and Moebius syndromes who were having difficulty in social relationships and collaborating with others used Paro, and Paro was found to alleviate problems in social relations, attachment and engagement and to stimulate sensorial exploration [23].

Researchers from Johns Hopkins School of Medicine developed a video game featuring an animated dolphin called 'Bandit'. The game was developed for stroke patients to improve their physical function by enabling them to control the dolphin using an exoskeleton robot [35]. Patients use a wide range of joint movements to lead the dolphin to move and eat in a playful immersive environment. As of this article, there are no academic publications on the acceptability or effectiveness of Bandit in stroke rehabilitation.

Paro the seal and Bandit the dolphin were designed for healthcare contexts and can be included in our study alongside Tamagotchi and ACs in the forms of a dog and cat.

\subsection{Multiplayer games as social support in rehabilitation}

Social interaction can improve patients' performance and motivation in rehabilitation therapy, however there exists relatively little research on social multiplayer games for rehabilitation therapy. In this section we discuss research on social interaction in rehabilitation games.

Maier et al. [18] studied social interaction within virtual reality multiplayer games for stroke rehabilitation. The games used adaptive mapping to compensate for motor impairments and fostered social interaction between patients and other participants by enabling playing in pairs (with other participants or with the caregivers). Patients could interact with their social networks at home or online. Maier et al. found that patients and their social circle enjoyed the play experiences as well as the social interactions. Ballester et al. [50] also investigated social interactions via a multiplayer game mode in a virtual reality system for stroke rehabilitation. They found that patients improved their extension movements more when playing multiplayer games than when playing single player games because the multiplayer environments positively affected patients' social engagement, motivation and performance.

Givon et al. [51] studied the feasibility of video games in group settings for stroke rehabilitation. The authors compared a "video game group", which was asked to play Xbox Kinect, PlayStation and Nintendo Wii games in pairs, and a "traditional group", which was asked to perform a normal exercise program such as warm up, stretching, and picking up objects in groups, pairs or triads. The authors report there was high treatment compliance for both interventions and rehabilitation outcomes (functional improvements) were similar, but satisfaction was rated higher for the video-game group.

Human companionship in multiplayer games is beneficial for stroke patients however other players may not always be available for the needed duration of gameplay for therapeutic purposes and ACs may provide benefits for this purpose. In order to explore how ACs may be integrated into digital stroke rehabilitation, we conduct a pre-adoption study to ask: what is the initial likeability of ACs by older adults, and why? By 'why', we mean what aspects of ACs are salient when making likeability judgements.

\section{Methods}

\subsection{Research context}

This project took place in an institution that offers art and art therapy programmes. The programme visited was entitled: "Leading a Creative Life: Enabling people with stroke to express themselves creatively through visual arts practice." The authors and a research assistant visited the institution on a weekly basis for the art studio time dedicated to those with complex disabilities and those recovering from stroke. We first conducted a field study, where we observed three weekly 2.5 hour sessions of art-making. During the subsequent weeks the authors conducted one-on-one interviews that included virtual reality art-making. The interview on ACs followed the virtual reality experience. We report on the field and interview results elsewhere [52]. This paper reports on $\mathrm{AC}$ results only. Each AC interview lasted between several minutes up to 10 minutes. 


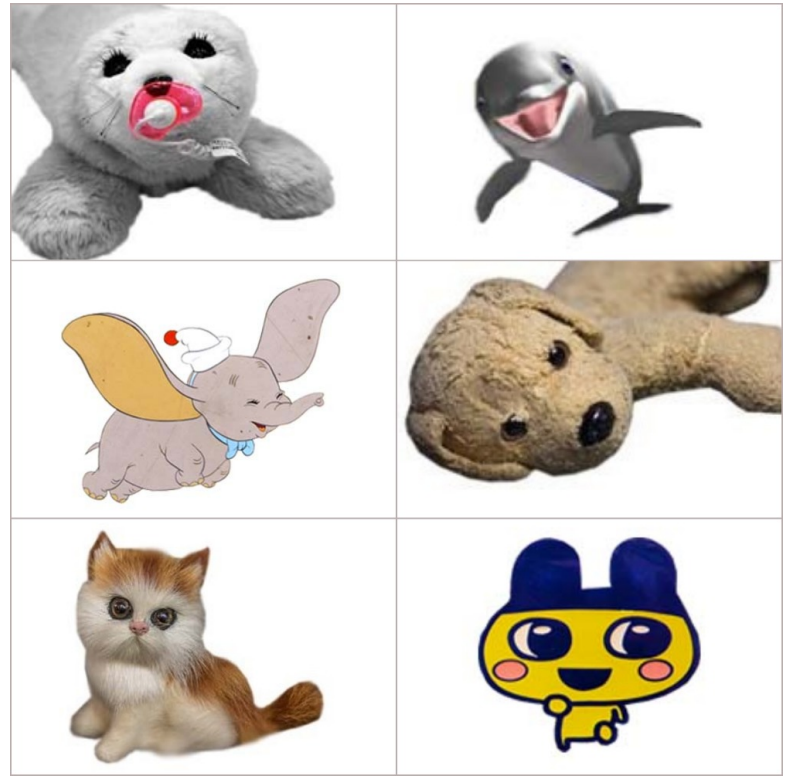

Figure 1. ACs in questionnaire. From top to bottom and left to right: Paro the Seal (1a) [53], Bandit the Dolphin (1b) [35], Dumbo (1c) [54], Dog (1d) [55], Cat (1e) [56], Tamagotchi (1f) [57]: (photos credit:

Paul Allais (1a), Courtesy of Max and Haley LLC

(1b), Tom Simpson (1c), DocChewbacca (1f))

Study protocols were approved by our institutional ethics committee. Thirteen participants provided written consent and one provided verbal consent. Eleven participated in the study on ACs.

\subsection{Protocol: interview, questionnaire and interactive experience}

We investigated initial impressions of ACs from literature: Tamagotchi, Paro the seal, Bandit the dolphin, a robot dog and a robot cat. As Paro, the cat and the dog are robots and Tamagotchi and Bandit are animated characters, we include one more animated ACs to balance the set: Dumbo the elephant, a widely known character from Disney's 1941 classic movie, Dumbo. Another advantage of Dumbo was that Dumbo's ability to fly would enable Dumbo to traverse vertical space in a virtual 3D environment, which may provide added benefit to stroke rehabilitation by encouraging a wide range of upper-limb movements.

We take an exploratory qualitative approach as this is the first study to examine ACs in a stroke rehabilitation context. As it is the first study with a relatively small sample, we opt for a thematic analysis approach [58] which provides themes from qualitative data. We frame the questions of first impressions in terms of likeability on a Likert scale, as this will be an easy to understand framing for participants. Our aim was to encourage them to focus on how they themselves liked the ACs, and to voice their own thoughts, feelings and impressions.

We conducted semi-structured interviews while participants looked at depictions of ACs on a printed questionnaire. The interviews were conducted in the art studio. We placed the printed questionnaire (Figure 1) ${ }^{1}$ on the table that we looked at together with the participant.

For each $\mathrm{AC}$ on the questionnaire, we asked: "For each character, how likeable is it on a scale from one to five, from one being not likeable to five being likeable?" "Also, please tell me how you feel about it. Can you imagine interacting with it? Why or, why not?" Additionally, we asked for any other thoughts or comments. Our final question for this portion was to ask participants which was their favourite among all the ACs.

After the questionnaire, we placed the iPad on the table to interact with the Tamagotchi game. We started by asking participants: "Have you ever played computer games / other games such as PlayStation, Nintendo Wii, Xbox Kinect before? What did you think of them?". We continued by saying: "Lets take a look at something else now: a game with a character named Tamagotchi. As you use it, let me know what you are thinking and feeling. Feel free to interact with the character". After participants interacted with it, we asked: "What did you think about it?"

The sessions were video recorded and transcribed for analysis [58]. Quantitative responses and themes are discussed next.

\subsection{Participants}

Our study included adults who had a stroke and were enrolled in the art programme. Some had comorbidities such as early-stage Parkinsons disease and mild dementia. Seven males and four females aged 55 - 79 years old participated. Table 1 provides demographic details for the participants.

\footnotetext{
${ }^{1}$ Due to copyright, Figure 1 presents similar images to the questionnaire; they are images that we have permission to reproduce. Original images from the questionnaire can be found at: 1) Paro (https://www.geospatialworld.net/blogs/robotic-seal-astronaut s-tackle-stress/),

2) Bandit (https://www.baltimoresun.com/health/bs-hs-dolphin-video -game-hopkins-20141015-story.html),

3) Dumbo (http://mickeyblog.com/2018/09/19/10-fun-facts-know-du $\mathrm{mbo} /)$,

4) Dog (https://github.com/VRArtforStroke/VR_Art_for_Stroke/blob/ master/local\%20copy.rar),

5) Cat (https://www.amazon.com/Joy-All-Silver-White-Mitts/dp/B01 7JQQ00Q),

6) Tamagotchi (https://tamagotchi.fandom.com/wiki/Adult_stage)
} 


\begin{tabular}{|c|c|c|c|}
\hline Participant & Gender & Age & $\begin{array}{c}\text { Functional } \\
\text { Hands }\end{array}$ \\
\hline P1 & F & 79 & Both \\
\hline P2 & F & 84 & Right \\
\hline P3 & F & 60 & Right \\
\hline P4 & M & n/a & Right \\
\hline P5 & M & 55 & Both \\
\hline P6 & F & 73 & Both \\
\hline P7 & M & 60 & Both \\
\hline P8 & M & 75 & Left \\
\hline P9 & M & 78 & Both \\
\hline P10 & M & 55 & Right \\
\hline P11 & M & 73 & Both \\
\hline
\end{tabular}

Table 1. Participants' demographic details

\section{Findings}

In this section, we first discuss the quantitative results of the questionnaires on $\mathrm{ACs}$, followed by the qualitative themes from the interviews and the themes arising from the Tamagotchi interactive experience: familiarity and having expectations for interaction.

\subsection{Companion likeability}

Participants rated the Dog and Cat as most likeable, with both positive and neutral ratings. Dumbo, Seal and Dolphin had similar averages but were more controversial, as they received negative ratings as well as positive scores. Tamagotchi was the least likeable. We conducted a repeated measures ANCOVA with age as a covariate. We found a significant difference between companions (Figure 2). Age was not significant ( $p>.05)$. A post-hoc test found that the Tamagotchi was significantly less liked than other ACs $(\mathrm{p}<.01)$, but that other ACs did not differ significantly from each other.

Next we discuss themes which emerged from what the participants said as they made their judgments.

\subsection{Familiarity and prior liking of creatures}

Participants shared that they liked a particular AC simply because they were familiar with the animal and already liked it (i.e., before taking part in the study) or because of the animal's 'playfulness' and other (assumed) characteristics. We noted that participants commented on the type of animal rather than the specific depiction. P2 said that she loves dogs and cats, without any further comments on why. P3, P4 and P6 chose the Dog as the most likeable and they each said that they like dogs. P9 picked Dumbo as his most likeable companion and he said that, "I really like Dumbo" without any further comments. P11 chose Dolphin as

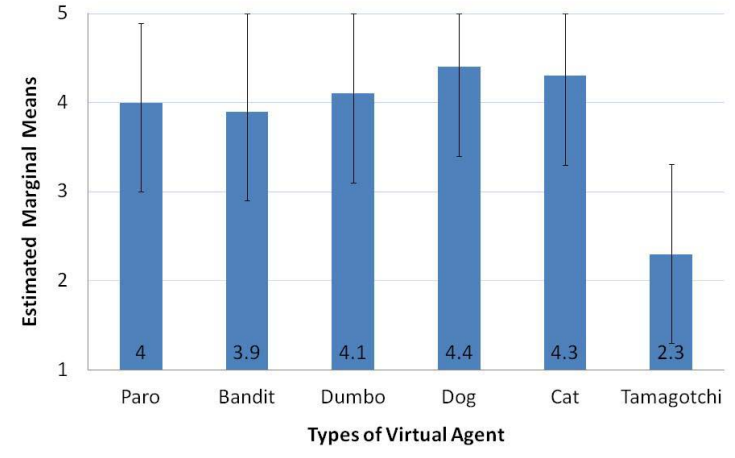

Figure 2. Mean ratings of ACs' likeability on a scale from 1 (not likeable) to 5 (likeable)

his most likeable companion, but he also mentioned that he likes dogs and picked Dog as his second choice along with Dumbo, Dog and Cat. P10 picked Cat as most likeable without further comment. P3 put Tamagotchi as her last choice because she was unfamiliar with it: "I don't understand what it is, if I don't understand what is it, I don't know if I like it or not." One might expect a neutral rating with that sentiment, however, P3 rated Tamagotchi as unlikeable at the endpoint of the scale.

The companions brought about general beliefs and reminiscence of personal memories in several participants. P3 chose the Seal as highly likeable and said, "they're often in the circus with balls." She explained her liking of the dolphin with "dolphins save people's lives." She rated Cat as highly likeable and she commented, "I don't like cats. They are playful and beautiful." P3 seemed to be ambivalent as she stated that she did not like cats in general, but thought of them in positive ways such as being playful and beautiful. P3 shared memories of a dog she used to have: a "small tiny little named Pickles. Pickles just jumped and ran against those dogs [German Shepherds], she just wanted to show she is the true $\operatorname{dog} .$. . She ran against those two huge dog like a monster. My sister said just run away. My sister was so proud of our dog." P10 also shared personal memories of his pets over his lifetime: "I got my lovely one Maliburung [name of pet bird]. [...] We've got two kittens. After that when it got bigger, one died... He was really friendly. He really loved me. He talked to me all the time. [...] I've got two cats and one dog. The dog, the big one, [...] he is sad on the inside. Very very sad on the inside. And I've got another cat. I've got two: one is black, one is white...". We saw that the representations of the social companions were able to trigger strong reminders and memories of previous 
experiences.

P1, P2, P3, P7, P8 and P10 asserted that they like pets. P8 mentioned that he likes pets such as cats, puppies and dogs, but he would be sad and disappointed if he lost his pet one day if he had one. P8 said: "Well, I can't handle them the way I am. But, I like cats, puppies, dogs. If I can have one, I would be disappointed to quit one". P6 and P11 noted that they did not like pets (though we note that P6 chose the Dog as the most likeable companion). P11 explained: "I don't like them but I tolerate it. My wife likes it. So for her sake, I look after them, I train them. They are good source of stress relief and more obedient than children." Note that P11 chose the Dolphin as his favourite, which is inline with his existing dislike of common pets.

We expected that participants may focus on the visual appeal of the ACs, such as overall cuteness or appeal of the faces of the creatures, however, only one participant explicitly mentioned cuteness as a factor for likeability. P1 said: "Dumbo is cute, I could get some for my grandchildren. I like him a lot. I like its face."

Next we turn to a theme related to familiarity: the ability to imagine interaction with the AC.

\subsection{Expectations of imagined interactions}

The ability to imagine interacting with the AC came up as a salient factor for participants. In commenting on the Dolphin, P5 said: "Nah, I find it hard to interact with it because it looks cartoonish." He continued: "I would probably interact with that one (Dog). The hardest one would be this one (Dolphin)." It is not completely clear why the Dog would be easier, but we propose that it may be jointly due to familiarity and ease of imagining how it would interact.

P6 stated that she could imagine interacting with all the companions. After some hesitation, she stated that she did like to take care of real pets and she did not like to play the Tamagotchi game. P11 picked Tamagotchi as the least favourite because he commented that Tamagotchi is a cartoon character. We cautiously suggest an interpretation of the salience of the cartoon nature of the companion representation. We believe that our participants may have had more difficulty imagining interacting with a cartoon representation as it would mean digital interaction, whereas the 'toy' representations enabled clearer expectations for interaction, such as real, physical interaction rather than digital interaction.

\subsection{Tamagotchi interactive experience}

Participants were invited to interact with a Tamagotchi, using a tablet with a mass-market
Tamagotchi game.

Most participants commented that they did not enjoy the interaction, although we observed that for the duration of the experience most continued interacting with the Tamagotchi character by tapping the screen. P2 was an exception who preferred to discontinue the session. P1 explained her dislike as "It drives me crazy. My son played a long time ago". P8 said: "I wouldn't go out of my way (for) this game". P5 explained that he did not like to play the game as he did not like to take care of pets because of having had a bad experience with a cat. Most participants overall disliked the interaction but some shared caveats. P10 mentioned that he did not like to play games but he said he would like to play if there were a virtual pet in a virtual art game. P11 stated that he did not like to play games but he plays with his granddaughter as a way of keeping interaction going between him and his granddaughter.

P3, P4 and P9 stated they liked interacting with a Tamagotchi. P9 said: "it's cool. Have a lot of fun with that". Data was missing from one participant due to a lack of internet connectivity during the session.

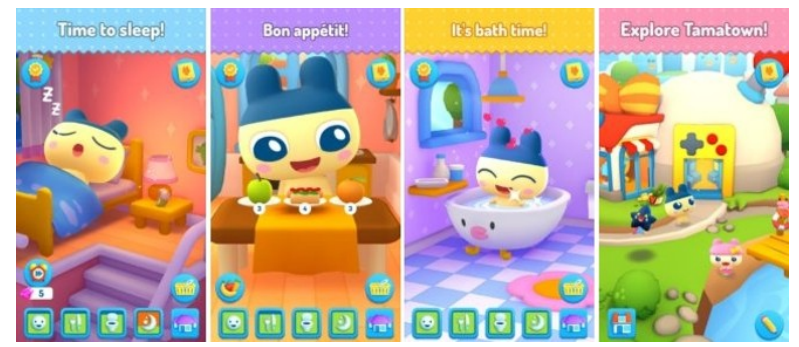

Figure 3. Tamagotchi interactive experience [59] (photo credit: mobilescout.com)

\section{Discussion}

Within an art rehabilitation studio during a creative stroke rehabilitation programme, we conducted 11 interviews on first impressions of artificial companion agents (ACs) using depictions and gameplay. We selected a range of ACs based on existing literature and mass-market companions: a robot cat, dog and seal (Paro) and an animated dolphin (Bandit), Dumbo and Tamagotchi. This investigation is an exploratory study to see what was salient to stroke survivors as they discussed the likeability of these various creatures. Better understanding perceptions of ACs may help designers incorporate them into stroke rehabilitation technologies (e.g., [60, 61, 62]).

Thematic analysis indicated that the familiarity mattered: familiarity was the strongest theme that emerged related to the likeability of the ACs. The most 
common animals, dogs and cats, reminded participants of previous interactions and previous pets. Paro the seal, Bandit the dolphin and Dumbo were less 'familiar' animals, but seals and dolphins are real animals. Elephants are also real, however, the flying aspect of Dumbo makes it less real. Tamagotchi was a likely unfamiliar and not real animal. Familiarity triggered reminiscence, where several participants spontaneously shared stories of interactions with pets or animals. Tamagotchi was found the least likeable and many participants explained that they were not familiar with the character and so it had less appeal.

Related to familiarity is the ability to imagine how one would interact with the ACs. Participants' ability to imagine interacting with the animals was the second most prevalent theme in our interactions. The robot or 'toy' representation may have helped participants to imagine how to interact with the $\mathrm{AC}$, and a digital cartoon representation appeared to make it more difficult to imagine interaction. This was exacerbated with Tamagotchi, as some participants could not imagine how it would act nor how to interact with it. Interestingly, even as participants gained experience in interacting with Tamagotchi, this did not lead to display of liking and in fact most repeated that they disliked it. As we found that ability to imagine interactions was a factor in likeability, it is possible that as participants learned how to interact with Tamagotchi, they may start to like it more but this was not the case. Our experience was relatively short, under 10 minutes, and so it may be that this could happen with more exposure and more interaction.

Familiarity has emerged as important in another game related study for older adults. Souders et al. conducted a 10 day study of videogaming by older adults where they compared two games with competitive and cooperative multiplayer interactions [63]. In their discussion, they note that their hypotheses were not validated, and that the differences in familiarly of the two games seemed to play a more important role than whether the participants cooperated or competed. Familiarity may play a more important role for older adults than with other age groups. We recommend that other researchers take care to note perceived familiarity of various aspects of their digital games and interactions, especially when working with older adults.

When planning our research, we did not anticipate that Tamagotchi would be significantly less likeable than other ACs. We included the interactive experience with Tamagotchi as it would simulate a digital companion. It was a useful exercise as it indicated that knowledge of interaction may not immediately increase liking. There are several reasons that the interaction with
Tamagotchi may not have been appealing. Older adults may have perceived that the game was for children and may have found the use of it infantilizing. Or, they may have simply not connected to the game due to different user needs based on their stage in life. Sherry et al. found that developmental stages affected reasons for playing, preferences for games and length of gameplay, with comparisons made between pre-adolescents, adolescents and young adults [64]. It may be that the Tamagotchi was not designed to meet the needs of older adults and thus is not appealing.

Our protocol did not explicitly ask older adults whether they perceived that the ACs were useful. A study on artifical pets claims that uselessness is a core quality for the success of artificial pets [65].

We expected that visual attractiveness or cuteness would play a larger role in likeability, but this was only mentioned by one participant. Lovot is described as being designed to have a cute face to foster emotional attachment [41]. Tamagotchi was popular among children because of its cuteness [66]. In our study, cuteness did not appear to strongly influence older adults as they focused more on familiarity.

Factors from Luh et al.'s companionship scale [45] can be related to the ones we identified, i.e., familiarity and expectations. Familiarity is associated with a higher attachment and is a precondition of trust [67]. Satisfaction is dependent on the gap between expectations and reality [68] in Expectation Confirmation Theory. For a familiar animal such as cat or dog, the gap between expectations and actual interactions is likely to be small, which might contribute to these ACs being preferred.

This research has several limitations. Our sample size was small. We opted to select companions that had existing research (Paro and Bandit) instead of choosing companions that could be presented in a digital or virtual environment. We believe that participants would still have focused on familiarity, but the theme on the ability to imagine interactions may have changed with the ability to interact. From including the interactive experience with Tamagotchi, the ability to interact for a few minutes may not significantly shift first impressions. Another limitation of including static images is the quality of the photos and placement of the companion. The photo may show the companion as more active or passive, which may have influenced our participants, even though they did not comment directly on those aspects. Familiarity was a salient characteristic to our participants, however, including more participants may have yielded a wider set of themes. We encourage future research to investigate this.

The mix of quantitative ratings and qualitative 
feedback was useful because we observed divergences between how participants rated companions, and how they verbally selected the one companion that they liked the most (in response to our verbal prompt). We found that most of our participants rated several companions with the same numerical rating, but easily identified the companion that they preferred above the others. Six participants (P3, P4, P5, P8, P9, P10) had more than one $\mathrm{AC}$ rated 5 on a 5 point scale of likeability, and gave verbal responses to specify which was the most likeable. None commented on how their preferences related to the ratings.

ACs may provide social support, but may also be beneficial by facilitating social support from others. For instance, older adults enjoy Paro and can build emotional attachment with it, especially in the presence of caregivers who also interact with it [69]. In our study, participants felt familiar with seals in general, which contributed to their liking of Paro. Familiarity was the driver of liking and familiar animals triggered reminiscence. It may be that familiarity with real pets drives likeability of artificial pets which drives adoption of the artificial pets by the elderly and people with disabilities. We recommend that future work explore if and why relationships between these concepts might exist and how this influences the decision to adopt ACs in the form of virtual pets.

We believe that these results are important to consider when designing companions because structured reminiscences programmes support cognition, mood and general function in older populations with dementia [70]. Rehabilitation system designers who are selecting ACs have the opportunity to select a familiar animal, which may provide additional rehabilitation support.

\section{Conclusion}

Artificial companions such as robot pets have been shown to be beneficial for elderly and people with disabilities by alleviating social isolation. In our study with stroke survivors, we found that companions' likeability differed with familiarity including existing liking of the animal, having personal memories from previous or current pets, and having expectation on how to interact with them. Selecting a familiar companion that supports reminiscence is an important opportunity when selecting ACs as part of the rehabilitation and healthcare among the elderly.

\section{Acknowledgement}

We thank our participants and the staff of Mapura Studio for their support of this research. The user study was approved by University of Auckland Human Participants Ethics Committee Reference number 021606. We thank our anonymous reviewers for their insights and recommendations.

\section{References}

[1] N. Bowles, "Human contact is now a luxury good." https://www.nytimes.com/2019/03/23/ sunday-review/human-contact-luxuryscreens.html Accessed: May 2019.

[2] "What is stroke?." http://www.stroke.org/ understand-stroke/what-stroke Accessed: June 2017.

[3] V. R. Venna and L. D. McCullough, "Role of social factors on cell death, cerebral plasticity and recovery after stroke," Metabolic Brain Disease, vol. 30, no. 2, pp. 497-506, 2015.

[4] Y. Chen, K. Abel, J. Janecek, Y. Chen, K. Zheng, and S. Cramer, "Home-based technologies for stroke rehabilitation: A systematic review," International Journal of Medical Informatics, vol. 123, pp. 11-22, 3 2019.

[5] J. Langan, H. Subryan, I. Nwogu, and L. Cavuoto, "Reported use of technology in stroke rehabilitation by physical and occupational therapists," Disability and Rehabilitation: Assistive Technology, vol. 13, no. 7, pp. 641-647, 2018.

[6] J.-H. Sun, L. Tan, and J.-T. Yu, "Post-stroke cognitive impairment: epidemiology, mechanisms and management," Annals of Translational Medicine, vol. 2, no. 8,2014 .

[7] B. French, L. H. Thomas, M. J. Leathley, C. J. Sutton, J. McAdam, A. Forster, P. Langhorne, C. I. Price, A. Walker, and C. L. Watkins, "Repetitive task training for improving functional ability after stroke," Stroke, vol. 40, no. 4, pp. e98-e99, 2009.

[8] J. W. Burke, M. McNeill, D. Charles, P. J. Morrow, J. Crosbie, and S. McDonough, "Augmented reality games for upper-limb stroke rehabilitation," in 2010 Second International Conference on Games and Virtual Worlds for Serious Applications, pp. 75-78, IEEE, 2010.

[9] S.-C. Yeh, W.-Y. Hwang, T.-C. Huang, W.-K. Liu, Y.-T. Chen, and Y.-P. Hung, "A study for the application of body sensing in assisted rehabilitation training," in 2012 International Symposium on Computer, Consumer and Control, pp. 922-925, IEEE, 2012.

[10] G. Burdea, "Keynote address: Virtual rehabilitation-benefits and challenges," in $1 s t$ International Workshop on Virtual Reality Rehabilitation (Mental Health, Neurological, Physical, Vocational) VRMHR, vol. 2002, sn, 2002.

[11] S. F. Bassett, "The assessment of patient adherence to physiotherapy rehabilitation," New Zealand Journal of Physiotherapy, vol. 31, no. 2, pp. 60-66, 2003.

[12] T. A. Türkbey, Ş. Kutlay, and H. Gök, "Clinical feasibility of xbox kinect tm training for stroke rehabilitation: A single-blind randomized controlled pilot study," Journal of Rehabilitation Medicine, vol. 49, no. 1, pp. 22-29, 2017.

[13] B. Lange, C.-Y. Chang, E. Suma, B. Newman, A. S. Rizzo, and M. Bolas, "Development and evaluation of 
low cost game-based balance rehabilitation tool using the microsoft kinect sensor," in Engineering in Medicine and Biology Society, EMBC, 2011 Annual International Conference of the IEEE, pp. 1831-1834, IEEE, 2011.

[14] G. N. Lewis, C. Woods, J. A. Rosie, and K. M. Mcpherson, "Virtual reality games for rehabilitation of people with stroke: perspectives from the users," Disability and Rehabilitation: Assistive Technology, vol. 6, no. 5, pp. 453-463, 2011.

[15] M. Goršič, I. Cikajlo, N. Goljar, and D. Novak, "A multisession evaluation of an adaptive competitive arm rehabilitation game," Journal of Neuroengineering and Rehabilitation, vol. 14, no. 1, p. 128, 2017.

[16] Y.-X. Hung, P.-C. Huang, K.-T. Chen, and W.-C. Chu, "What do stroke patients look for in game-based rehabilitation: a survey study," Medicine, vol. 95, no. 11, 2016.

[17] L. A. Hale, "Community-based or home-based stroke rehabilitation: confusion or common sense?," New Zealand Journal of Physiotherapy, vol. 32, pp. 131-139, 2004.

[18] M. Maier, B. R. Ballester, E. Duarte, A. Duff, and P. F. Verschure, "Social integration of stroke patients through the multiplayer rehabilitation gaming system," in International Conference on Serious Games, pp. 100-114, Springer, 2014.

[19] K. Jack, S. M. McLean, J. K. Moffett, and E. Gardiner, "Barriers to treatment adherence in physiotherapy outpatient clinics: a systematic review," Manual Therapy, vol. 15, no. 3, pp. 220-228, 2010.

[20] V. Amati, S. Meggiolaro, G. Rivellini, and S. Zaccarin, "Social relations and life satisfaction: the role of friends," Genus, vol. 74, no. 1, p. 7, 2018.

[21] M. Van der Horst and H. Coffé, "How friendship network characteristics influence subjective well-being," Social Indicators Research, vol. 107, no. 3, pp. 509-529, 2012.

[22] A. M. Beck and N. M. Meyers, "Health enhancement and companion animal ownership," Annual Review of Public Health, vol. 17, no. 1, pp. 247-257, 1996.

[23] P. Marti, A. Pollini, A. Rullo, and T. Shibata, "Engaging with artificial pets," in Proceedings of the 2005 Annual Conference on European Association of Cognitive Ergonomics, pp. 99-106, University of Athens, 2005.

[24] H. Robinson, B. MacDonald, N. Kerse, and E. Broadbent, "The psychosocial effects of a companion robot: a randomized controlled trial," Journal of the American Medical Directors Association, vol. 14, no. 9, pp. 661-667, 2013.

[25] J. F. Hoorn, "From lonely to resilient through humanoid robots: Building a new framework of resilience," Journal of Robotics, vol. 2018, 2018.

[26] G. Luong, S. T. Charles, and K. L. Fingerman, "Better with age: Social relationships across adulthood," Journal of Social and Personal Relationships, vol. 28, no. 1, pp. 9-23, 2011.

[27] C. M. Holanda, F. L. De Andrade, M. A. Bezerra, J. P. Nascimento, R. Neves, S. B. Alves, and K. S. Ribeiro, "Support networks and people with physical disabilities: social inclusion and access to health services," Ciencia \& Saude Coletiva, vol. 20, pp. 175-184, 2015.

[28] A. N. Amado, R. J. Stancliffe, M. McCarron, and P. McCallion, "Social inclusion and community participation of individuals with intellectual/developmental disabilities," Intellectual and Developmental Disabilities, vol. 51, no. 5, pp. 360-375, 2013.

[29] Y. Wilks, Close engagements with artificial companions: key social, psychological, ethical and design issues, vol. 8. John Benjamins Publishing, 2010.

[30] O. Mival, S. Cringean, and D. Benyon, "Personification technologies: Developing artificial companions for older people," CHI Fringe, Austria, 2004.

[31] M. Heerink, B. Kröse, V. Evers, and B. Wielinga, "Assessing acceptance of assistive social agent technology by older adults: the almere model," International Journal of Social Robotics, vol. 2, no. 4, pp. 361-375, 2010.

[32] S. Kriglstein and G. Wallner, "Homie: an artificial companion for elderly people," in CHI'05 Extended Abstracts on Human Factors in Computing Systems, pp. 2094-2098, ACM, 2005.

[33] A. Liang, I. Piroth, H. Robinson, B. Macdonald, M. Fisher, U. Nater, N. Skoluda, and E. Broadbent, "A pilot randomized trial of a companion robot for people with dementia living in the community," Journal of the American Medical Directors Association, vol. 18, 06 2017.

[34] Y.-F. Tsai and D. Kaufman, "Interacting with a computer-simulated pet: Factors influencing children's humane attitudes and empathy," Journal of Educational Computing Research, vol. 51, no. 2, pp. 145-161, 2014.

[35] A. Karidis, "Hopkins' new video game may pave way for stroke therapy." https:// www.baltimoresun.com/health/bs-hsdolphin-video-game-hopkins-20141015story.html Accessed: June 2019.

[36] G. Melson, A. Beck, and B. Friedman, "Robotic pets in human lives: Implications for the human-animal bond and for human relationships with personified technologies," Journal of Social Issues - J SOC ISSUES, vol. 65, pp. 545-567, 092009.

[37] H. Robinson, E. Broadbent, and B. Macdonald, "Group sessions with paro in a nursing home: Structure, observations and interviews: Sessions with paro in a nursing home," Australasian Journal on Ageing, vol. 35, 062015.

[38] S. Coghlan, J. Waycott, B. B. Neves, and F. Vetere, "Using robot pets instead of companion animals for older people: A case of 'reinventing the wheel'?," in Proceedings of the 30th Australian Conference on Computer-Human Interaction, OzCHI '18, (New York, NY, USA), pp. 172-183, ACM, 2018.

[39] C. Lutz and A. Tamo, "Privacy and healthcare robots an ant analysis," in Proceedings of the We Robot Conference 2016, Apr. 2016.

[40] M. Coeckelbergh, "Why care about robots? empathy, moral standing, and the language of suffering," Kairos. Journal of Philosophy \& Science, vol. 20, no. 1, pp. 142-158, 2018.

[41] D. Lee, "Lovot is the first robot i can see myself getting emotionally attached to." https: //www.theverge.com/2019/1/10/18176002/ lovot-groovex-robot-emotionalattachment-ces-2019. Accessed: June 2019.

[42] M. Dragone, T. Holz, and G. M. O'Hare, "Using mixed reality agents as social interfaces for robots," in $R O-M A N$ 
2007-The 16th IEEE International Symposium on Robot and Human Interactive Communication, pp. 1161-1166, IEEE, 2007.

[43] C. L. Lisetti and E. Wagner, "Mental health promotion with animated characters: Exploring issues and potential.," in AAAI Spring Symposium: Emotion, Personality, and Social Behavior, pp. 72-79, 2008.

[44] D. R. Billings, K. E. Schaefer, J. Y. C. Chen, and P. A. Hancock, "Human-robot interaction: Developing trust in robots," in 2012 7th ACM/IEEE International Conference on Human-Robot Interaction (HRI), pp. 109-110, March 2012.

[45] D.-B. Luh, E. C. Li, and Y.-J. Kao, "The Development of a Companionship Scale for Artificial Pets," Interacting with Computers, vol. 27, pp. 189-201, 012014.

[46] R. Hawkins, J. Williams, et al., "Childhood attachment to pets: Associations between pet attachment, attitudes to animals, compassion, and humane behaviour," International Journal of Environmental Research and Public Health, vol. 14, no. 5, p. 490, 2017.

[47] L. Pu, W. Moyle, C. Jones, and M. Todorovic, "The Effectiveness of Social Robots for Older Adults: A Systematic Review and Meta-Analysis of Randomized Controlled Studies," The Gerontologist, vol. 59, pp. e37-e51, 062018.

[48] YellRobot, "Zora robot acts as companion for dementia patients." https://yellrobot.com/zorarobot-companion-dementia-nursinghealthcare/ Accessed: June 2019.

[49] C. Huisman and H. Kort, "Two-year use of care robot zora in dutch nursing homes: An evaluation study," in Healthcare, vol. 7, p. 31, Multidisciplinary Digital Publishing Institute, 2019.

[50] B. R. Ballester, S. B. i Badia, and P. F. Verschure, "Including social interaction in stroke vr-based motor rehabilitation enhances performance: a pilot study," Presence, vol. 21, no. 4, pp. 490-501, 2012.

[51] N. Givon, G. Zeilig, H. Weingarden, and D. Rand, "Video-games used in a group setting is feasible and effective to improve indicators of physical activity in individuals with chronic stroke: a randomized controlled trial," Clinical Rehabilitation, vol. 30, no. 4, pp. 383-392, 2016.

[52] M. Alex, B. C. Wünsche, and D. Lottridge, "Virtual reality art-making for stroke rehabilitation: Field study and technology probe," Under preparation. [53] P. Allais, "Paro the robo-seal." https:
// www.flickr.com/photos/hokutosuisse/ 4777167904 Accessed: June 2019.

[54] T. Simpson, "1955 mickey mouse club circus sideshow poster - dumbo." https://www.flickr.com/ photos/gameraboy/30261694628. Accessed: June 2019.

[55] Pxhere, "dog." https://pxhere.com/en/photo/ 658829. Accessed: June 2019.

[56] Blickpixel, "Cat kitten toys plush." https : //pixabay.com/photos/cat-kitten-toysplush-soft-toy-512688/ Accessed: June 2019.

[57] DocChewbacca, "Tamagotchi keyholders." https://www.flickr.com/photos/st3f4n/ 2848658859 Accessed: June 2019.
[58] V. Braun and V. Clarke, "Using thematic analysis in psychology," Qualitative Research in Psychology, vol. 3, no. 2, pp. 77-101, 2006.

[59] Mobilescout, "My tamagotchi forever." https: //www.mobilescout.com/android/news/ n104482/my-tamagotchi-forever-gameavailable-download.html. Accessed: June 2019.

[60] C. Robertson, L. Vink, H. Regenbrecht, C. Lutteroth, and B. C. Wünsche, "Mixed reality kinect mirror box for stroke rehabilitation," in 28th International Conference on Image and Vision Computing New Zealand (IVCNZ 2013), pp. 231-235, Nov 2013.

[61] N. Nordin, S. Q. Xie, and B. C. Wünsche, "Assessment of movement quality in robot- assisted upper limb rehabilitation after stroke: a review," Journal of Neuroengineering and Rehabilitation, vol. 11, no. 1, p. 23, 2014.

[62] M. Alex, C.-Y. Chen, and B. C. Wünsche, "A review of sensor devices in stroke rehabilitation," in International Conference on Image and Vision Computing New Zealand (IVCNZ 2017), pp. 1-6, Dec 2017.

[63] D. J. Souders, W. R. Boot, N. Charness, and J. H. Moxley, "Older adult video game preferences in practice: investigating the effects of competing or cooperating," Games and Culture, vol. 11, no. 1-2, pp. 170-200, 2016.

[64] J. Sherry, R. Desouza, B. Greenberg, and K. Lachlan, "Relationship between developmental stages and video game uses and gratifications, game preference and amount of time spent in play," in Annual Conference of the International Communication Association, San Diego, Calif, 2003.

[65] F. Kaplan, "Free creatures: The role of uselessness in the design of artificial pets," in Proceedings of the 1st Edutainment Robotics Workshop, pp. 45-47, Citeseer, 2000.

[66] L. Lawton, "Taken by the tamagotchi: How a toy changed the perspective on mobile technology," The iJournal: Graduate Student Journal of the Faculty of Information, vol. 2, no. 2, 2017.

[67] D. Gefen, "E-commerce: the role of familiarity and trust," Omega, vol. 28, no. 6, pp. $725-737,2000$.

[68] R. L. Oliver, "Effect of expectation and disconfirmation on postexposure product evaluations: An alternative interpretation.," Journal of Applied Psychology, vol. 62, no. 4, p. 480, 1977.

[69] C. D. Kidd, W. Taggart, and S. Turkle, "A sociable robot to encourage social interaction among the elderly," in Proceedings 2006 IEEE International Conference on Robotics and Automation, 2006. ICRA 2006., pp. 3972-3976, IEEE, 2006.

[70] B. Woods, L. O'Philbin, E. M. Farrell, A. E. Spector, and M. Orrell, "Reminiscence therapy for dementia," Cochrane Database of Systematic Reviews, no. 3, 2018. 